\title{
Leserforum
}

\section{On the Loading of a Stop-Loss Contract: a Correction on Extrapolation and two Stable Price Methods}

Werner Hürlimann (Winterthur)

\section{Correction}

In the short note by E. Kremer (1998), the factor 2 in the differential equation $\left(^{*}\right)$ and the extrapolation formula (**) should not appear. This invalidates the proposed rule of thumb, which is less good than the presented extrapolation. For the given example, on has in tabular form

\begin{tabular}{lllll}
\hline $\mathrm{P}_{2}$ & $\mathrm{~m}\left(\mathrm{P}_{2}\right)$ & $\mathrm{s}\left(\mathrm{P}_{2}\right)$ & $\mathrm{ss}$ & $\frac{1}{2}\left(\mathrm{ss}+\mathrm{s}\left(\mathrm{P}_{1}\right)\right)$ \\
\hline 1.7 & 0.1827 & 0.5762 & 0.5704 & 0.5364 \\
1.9 & 0.1496 & 0.5261 & 0.5255 & 0.5139 \\
2.1 & 0.1225 & 0.4795 & 0.4789 & 0.4906 \\
2.3 & 0.1003 & 0.4364 & 0.431 & 0.4667 \\
2.5 & 0.0821 & 0.3968 & 0.382 & 0.4422 \\
\hline
\end{tabular}

The extrapolation yields a slight underestimation, which can be accepted in view of the other inherent uncertainties in stop-loss calculations.

In Kremer's note there is no hint on how to get the unknown factor loading $\lambda$ in the formula $I(P)=\lambda \cdot s(P)$. In the recent (not yet published) research monograph Hürlimann (1997a), two distribution-free methods are considered. Our second method shows that, under certain circumstances, there is actually no need for a knowledge of the standard deviation of the stop-loss claim. In the first method, the standard deviation can be bounded by Schmitter's inequalities.

\section{General Stable Price Method}

Let $S$ represent the claim size of a line of insurance business over some fixed period, usually called risk, which is described by a random variable with known moments up to the $\mathrm{n}^{\text {th }}$ order, $\mathrm{n}=2,3,4, \ldots$, say $\mathrm{S} \in \mathrm{D}_{\mathrm{n}}$. By assumption, at least the mean $\mu$ and standard deviation $\sigma$ are known. Let $P=\mathrm{H}[\mathrm{S}]$ be the price of the risk, where $\mathrm{H}[\cdot]$ is a real probability functional from $D_{n}$ to $R_{+}$. The possible loss over the fixed period is described by the random variable $L=S-P$. It is often reasonable to set prices according to the stability criterion $\operatorname{Pr}(L>0)=F_{S}(P) \leq \varepsilon$, where $\varepsilon$ is a small prescribed positive number. It says that the probability of insolvability in a long position of this line of business is less than $\varepsilon$. In a situation of incomplete information like $S \in D_{n}$, stability is achieved provided

$$
\max _{S \in D_{\mathbf{n}}}\left\{F_{S}(P)\right\}=\varepsilon,
$$

which can be called distribution-free stability criterion. The solution $P$ to (2.1) will be called stable price. The stable price is determined according to a standard deviation principle $\mathrm{P}=\mathrm{H}_{\varepsilon}^{(\mathrm{n})}[\mathrm{S}]$ $=\mu+\theta_{\varepsilon}^{(n)} \cdot \sigma$, where the loading factor $\theta_{\varepsilon}^{(n)}$ is the $\varepsilon$-percentile obtained from

$$
F^{(n)}\left(\theta_{\varepsilon}^{(n)}\right)=\varepsilon,
$$

where $F^{(n)}(x)$ denotes the standard Chebyshev-Markov extremal distribution (when $n$ moments are known).

The dependence upon the portfolio size is very simple. For a portfolio of $\mathrm{N}$ independent and identically distributed risks $S_{i}$ with aggregate portfolio risk $S=\Sigma S_{i}$, the loading factor can be 
reduced by a factor of $(\sqrt{\mathrm{N}})^{-1}$. Indeed, let $P_{i}=\mu+\theta_{e, i}^{(n)} \cdot \sigma$ be the stable price of an individual risk $\mathrm{S}_{\mathrm{i}}$ with mean $\mu$ and standard deviation $\sigma$, where $\theta_{\varepsilon, \mathrm{i}}^{(\mathrm{n})}$ is an individual loading factor. Then the portfolio risk $S=\Sigma S_{i}$ has mean $N \cdot \mu$ and standard deviation $\sqrt{N} \cdot \sigma$. Let $P=N \cdot \mu+\theta_{z}^{(n)} \cdot \sqrt{N} \cdot \sigma$ be the portfolio stable price, where the portfolio loading factor $\theta_{e}^{(n)}$ is determined by (2.2). Since $\mathrm{P}=\Sigma \mathrm{P}_{\mathrm{i}}$ one must have $\theta_{\varepsilon, \mathrm{i}}^{(\mathrm{n})}=(\sqrt{\mathrm{N}})^{-1} \cdot \theta_{\varepsilon}^{(\mathrm{n})}$, which determines the individual loading factor in terms of the portfolio loading factor. In view of the general inequalities $F^{(n+1)}(x) \leq F^{(n)}(x)$, $\mathrm{n}=2,3,4, \ldots$, the loading factor. $\theta_{\varepsilon}^{(n)}$ is a decreasing function of $\mathrm{n}$, that is the stable price decreases when more and more information is available about the risk. Since $F^{(n)}(x)$ has been completely described for $n=2,3,4$, the corresponding stable prices can be determined, at least numerically, using a computer algebra system.

Applying the above pricing method to stop-loss risk $R=(S-d)_{+} \in D_{2}$, with $m(d)=E[R]$ the net stop-loss premium and $s(d)=\sqrt{\operatorname{Var}[R]}$ the standard deviation, one obtains with $\theta_{\varepsilon}^{(2)}=\sqrt{\frac{1-\varepsilon}{\varepsilon}}$ the
loaded stop-loss premium

$$
\mathrm{P}_{1}=\mathrm{m}(\mathrm{d})+\sqrt{\frac{1-\varepsilon}{N \cdot \varepsilon}} \cdot \mathrm{s}(\mathrm{d}) .
$$

In case the standard deviation is not known, it can be bounded by the so-called inequalities of Schmitier (see Hürlimann (1997b/c)):

$$
\begin{aligned}
s_{u}^{2}(d): & =\frac{1}{2}\left[\sigma^{2}-2 m(d) \bar{m}(d)-\sigma \sqrt{\sigma^{2}-4 m(d) \bar{m}(d)}\right] \\
& \leq s^{2}(d) \leq \\
s_{o}^{2}(d): & =\frac{1}{2}\left[\sigma^{2}-2 m(d) \bar{m}(d)+\sigma \sqrt{\sigma^{2}-4 m(d) \bar{m}(d)}\right]
\end{aligned}
$$

with $\overline{\mathrm{m}}(\mathrm{d})=\mathrm{d}-\mu+\mathrm{m}(\mathrm{d})$ the "conjugate" stop-loss transform. Inserted in (2.3) these bounds yield loaded stop-loss premiums $P_{1}^{u}$ and $P_{1}^{\circ}$ respectively.

\section{Stop-Loss Stable Price Method}

The general stable price method, developed in Section 2, can be applied to transformed risks of a line of business. Let us calculate the stable stop-loss prices of a stop-loss risk $S(d)=(S-d)_{+}$with deductible $d$ for a random variable $S \in D_{n}$ with known moments up to the $n^{\text {th }}$ order, $n=2,3,4, \ldots$, which is denoted by $P_{z}^{(n)}(d)$. The possible loss over a fixed period is described by the random variable $\mathrm{L}=\mathrm{S}-\mathrm{d}-\mathrm{P}_{e}^{(n)}(\mathrm{d})$. Then the distribution-free stability criterion

$$
\max _{S \in D_{\mathbf{n}}}\left\{F_{S}\left(d+P_{\varepsilon}^{(n)}(d)\right)\right\}=\varepsilon
$$

implies a stable stop-loss price of amount

$$
P_{\varepsilon}^{(n)}(d)=\theta_{\varepsilon}^{(n)} \cdot \sigma-(d-\mu) .
$$

Suppose now that stop-loss prices are set according to the standard deviation principle

$$
\mathrm{H}[(\mathrm{S}(\mathrm{d})]=\mathrm{m}(\mathrm{d})+\theta \cdot \mathrm{s}(\mathrm{d}) \text {. }
$$

Comparing (3.2) and (3.3) the standard deviation loading must be equal to

$$
\theta \cdot \mathrm{s}(\mathrm{d})=\theta_{\varepsilon}^{(\mathrm{n})} \cdot \sigma-(\mathrm{d}-\mu)-\mathrm{m}(\mathrm{d}) .
$$

For a portfolio of $\mathrm{N}$ independent and identically distributed stop-loss risks, the individual loading factor can be reduced by a factor of $(\sqrt{N})^{-1}$ as explained after formula (2.2). Therefore, it is 
reasonable to set stable stop-loss prices at the level

$$
H_{N}[X(d)]=\pi(d)+l_{N}(d)
$$

where the loading equals

$$
l_{N}(d)=\frac{1}{\sqrt{N}} \cdot\left(\theta_{\varepsilon}^{(n)} \cdot \sigma-(d-\mu)-m(d)\right) .
$$

One observes that the present method does not depend on the knowledge of the standard deviation of the stop-loss risk. However, it is more conservative because it views $L=S-d-P_{z}^{(n)}$ as possible loss and not $L=S(d)-P_{1}$ as in the first method. This difference in point of view requires an explanation. The second method corresponds to the strategy of a direct insurer, who desires to cover his claims above $d$ through reinsurance, and offers the insured a dividend or bonus of amount $(d-S)_{+}$. The amount $d+P_{e}^{(n)}$ is the total stable premium required to realize this insurance strategy, which turns out to be riskless for the direct insurer. In analogy to Table 1 in Hürlimann (1994), p. 179, one has the following accounting scheme:

\section{Income}

Stable premium

Reinsurance payment

$$
\begin{aligned}
& d+P_{\varepsilon}^{(n)} \\
& (S-d)_{+}
\end{aligned}
$$

\section{Outcome}

Claims payment

$S$

Guaranteed dividend

Reinsurance cost of strategy

$(d-S)_{+}$

$P_{\varepsilon}^{(n)}$

Net outcome

0

\section{Illustration}

For the academic exponential model considered by Kremer (1998) with $\mu=\sigma=1$, one obtains the quantities:

\begin{tabular}{lllll}
\hline $\mathrm{d}$ & $\mathrm{m}(\mathrm{d})$ & $\mathrm{s}(\mathrm{d})$ & $\mathrm{s}_{\mathrm{u}}(\mathrm{d})$ & $\mathrm{s}_{\mathrm{o}}(\mathrm{d})$ \\
\hline 1 & 0.3679 & 0.7749 & 0.1614 & 0.8386 \\
2 & 0.1353 & 0.5023 & 0.1896 & 0.8104 \\
3 & 0.0498 & 0.3116 & 0.1154 & 0.8846 \\
\hline
\end{tabular}

If only the information $\mu, \sigma, m\left(d_{1}\right), \ldots, m\left(d_{n}\right)$ is availabie, where $d_{1}<d_{2}<\cdots<d_{n}$, missing values can be obtained through extrapolation. For $m(d)$ use linear interpolation (exact for a random variable with a finite atomic support):

$$
m(d)=m\left(d_{m}\right)-\left(d-d_{m}\right) \cdot \frac{m\left(d_{m}\right)-m\left(d_{m+1}\right)}{d_{m+1}-d_{m}}, \quad d_{m} \leq d \leq d_{m+1}
$$

If $s\left(d_{1}\right), \ldots, s\left(d_{n}\right)$ are not known, use Schmitter's bounds (2.4), otherwise use Kremer's extrapolation formula. Calculation of the different stop-loss premiums, by fixing $\varepsilon=0.05$ and varying the portfolio size $\mathrm{N}$, yields the table: 


\begin{tabular}{llllll}
\hline & $N$ & $P_{1}$ & $P_{1}^{u}$ & $P_{1}^{0}$ & $P_{\varepsilon}^{(2)}$ \\
\hline$d=1$ & 10 & 1.436 & 0.5903 & 1.5238 & 1.63 \\
& 100 & 0.7056 & 0.4382 & 0.7334 & 0.767 \\
& 1000 & 0.4747 & 0.3901 & 0.4835 & 0.4941 \\
& 10000 & 0.4017 & 0.3749 & 0.4044 & 0.4078 \\
& 100000 & 0.3786 & 0.3701 & 0.3794 & 0.3805 \\
$d=2$ & 10 & 0.8278 & 0.3967 & 1.2524 & 1.1547 \\
& 100 & 0.3543 & 0.218 & 0.4886 & 0.4577 \\
& 1000 & 0.2046 & 0.1615 & 0.247 & 0.2373 \\
& 10000 & 0.1572 & 0.1436 & 0.1707 & 0.1676 \\
& 100000 & 0.1423 & 0.1379 & 0.1465 & 0.1455 \\
$\mathrm{~d}=3$ & 10 & 0.4793 & 0.2088 & 1.2692 & 0.78 \\
& 100 & 0.1856 & 0.1001 & 0.4354 & 0.2807 \\
& 1000 & 0.0927 & 0.0657 & 0.1717 & 0.1228 \\
& 10000 & 0.0634 & 0.0548 & 0.0883 & 0.0729 \\
& 100000 & 0.0541 & 0.0514 & 0.062 & 0.0571 \\
\hline
\end{tabular}

The portfolio size is the critical parameter. A small reinsurer with only 10 stop-loss contracts cannot be competitive on the reinsurance market. He requires at least the double stable stop-loss price when compared with reinsurers with more than 100 contracts. Schmitter's upper bound for the standard deviation yields good results for deductibles not far away from the mean, but seems too conservative otherwise.

\section{REFERENCES}

Hürlimann, W. (1994): Splitting risk and premium calculation. Bulletin of the Swiss Association of Actuaries, 167-197.

Hürlimann, W. (1997a): Extremal Moment Methods and Stochastic Orders. Applications in Actuarial Science. Revised monograph manuscript (1998).

Hürlimann, W. (1997b): An elementary unified approach to loss variance bounds. Bulletin of the Swiss Association of Actuaries, 73-88.

Hürlimann, W. (1997c): Fonctions extrémales et gain financier. Elemente der Mathematik 52, $152-168$.

Kremer, E. (1998): Extrapolating the Loading of a Stoploss Treaty. Blätter der Deutschen Gesellschaft für Versicherungsmathematik, Band XXIII, Heft 4, 623-624.

\section{Zusammenfassung}

Über den Sicherheitszuschlag eines Stop-Loss Vertrags:

Korrektur einer Extrapolation und zwei stabile Preismethoden

Eine Formel für die Extrapolation der Standardabweichung eines Stop-Loss Risikos wird korrigiert. Zwei stabile Preismethoden zur Ermittlung der unbekannten Brutto-Stop-Loss Prämien werden vorgestellt. Die zweite Methode ist nützlich im Zusammenhang mit risikolosen Strategien für Direktversicherer. Unsere Illustration identifiziert die Portefeuilles Größe als kritischer Parameter für Brutto-Stop-Loss Prämien.

\section{Summary}

On the Loading of a Stop-Loss Contract:

a Correction on Extrapolation and two Stable Price Methods

An extrapolation formula by Kremer for the standard deviation of a stop-loss claim is corrected. Two stable pricing methods to determine the unknown loaded stop-loss premiums are presented. The second method is useful in the context of riskless direct insurance strategies. Our illustration identifies the portfolio size as critical risk parameter for loaded stop-loss premiums. 